\title{
Representation of odd integers as the sum of one prime, two squares of primes and powers of 2
}

\author{
by
}

Hongze Li (Shanghai)

1. Introduction. Let

$$
\mathcal{A}=\{n: n \in \mathbb{N}, n \not \equiv 0(\bmod 2), n \not \equiv 2(\bmod 3)\} .
$$

In 1938 Hua [3] proved that almost all $n \in \mathcal{A}$ are representable as sums of two squares of primes and a $k$ th power of a prime for odd $k$,

$$
n=p_{1}^{2}+p_{2}^{2}+p_{3}^{k} .
$$

In 1999, Liu, Liu and Zhan [6] proved that every large odd integer $N$ can be written as a sum of one prime, two squares of primes and $k$ powers of 2 ,

$$
N=p_{1}^{2}+p_{2}^{2}+p_{3}+2^{\nu_{1}}+\cdots+2^{\nu_{k}} .
$$

In 2004, Liu [8] proved that $k=22000$ is acceptable in (1.2).

In this paper we shall prove the following result.

THEOREM. Every sufficiently large odd integer can be written as a sum of one prime, two squares of primes and 106 powers of 2.

The substantial improvement is due to two facts: firstly, we use the method of [1] and [7] to enlarge the major arcs; secondly, Heath-Brown and Puchta's estimation for the measure of exponential sums of powers of 2 (Lemma 3) gives a good control for the minor arcs.

2. Outline and preliminary results. To prove the Theorem, it suffices to estimate the number of solutions of the equation

$$
n=p_{1}^{2}+p_{2}^{2}+p_{3}+2^{\nu_{1}}+\cdots+2^{\nu_{k}} .
$$


Suppose $N$ is our main parameter, which we assume to be "sufficiently large". We write

$$
P=N^{1 / 6-\varepsilon}, \quad Q=N P^{-1} L^{-10}, \quad M=N L^{-9}, \quad L=\log _{2} N .
$$

We use $c$ and $\varepsilon$ to denote an absolute constant and a sufficiently small positive number, not necessarily the same at each occurrence.

The circle method, in the form we require, begins with the observation that

$$
\begin{aligned}
R(N) & :=\sum_{\substack{p_{1}^{2}+p_{2}^{2}+p_{3}+2^{\nu_{1}}+\cdots+2^{\nu_{k}=N} \\
M<p_{1}^{2}, p_{2}^{2}, p_{3} \leq N}}\left(\log p_{1}\right)\left(\log p_{2}\right)\left(\log p_{3}\right) \\
& =\int_{0}^{1} f^{2}(\alpha) g(\alpha) h^{k}(\alpha) e(-\alpha N) d \alpha,
\end{aligned}
$$

where we write $e(x)=\exp (2 \pi i x)$ and

$$
\begin{aligned}
& f(\alpha)=\sum_{M<p^{2} \leq N}(\log p) e\left(\alpha p^{2}\right), \quad g(\alpha)=\sum_{M<p \leq N}(\log p) e(\alpha p), \\
& h(\alpha)=\sum_{2^{\nu} \leq N} e\left(\alpha 2^{\nu}\right):=\sum_{\nu \leq L} e\left(\alpha 2^{\nu}\right) .
\end{aligned}
$$

By Dirichlet's lemma on rational approximation, each $\alpha \in[1 / Q, 1+1 / Q]$ can be written as

$$
\alpha=\frac{a}{q}+\beta, \quad|\beta| \leq \frac{1}{q Q},
$$

for some integers $a, q$ with $1 \leq a \leq q \leq Q,(a, q)=1$. Let

$$
\mathfrak{M}=\bigcup_{1 \leq q \leq P} \bigcup_{\substack{a=1 \\(a, q)=1}}^{q}\left[\frac{a}{q}-\frac{1}{q Q}, \frac{a}{q}+\frac{1}{q Q}\right] .
$$

These are the major arcs, and the minor arcs $\mathfrak{m}$ are given by

$$
\mathfrak{m}=\left[\frac{1}{Q}, 1+\frac{1}{Q}\right] \backslash \mathfrak{M} .
$$

Lemma 1 (Theorem 3 of [4] for $k=2$ ). Suppose that $\alpha$ is a real number and there exist $a \in \mathbb{Z}$ and $q \in \mathbb{N}$ satisfying

$$
1 \leq q \leq Y, \quad(a, q)=1, \quad|q \alpha-a|<Y^{-1},
$$

with $Y=X^{3 / 2}$. Then for any fixed $\varepsilon>0$ one has

$$
\sum_{X<p \leq 2 X}(\log p) e\left(\alpha p^{2}\right) \ll X^{7 / 8+\varepsilon}+\frac{q^{\varepsilon} X(\log X)^{c}}{\left(q+X^{2}|q \alpha-a|\right)^{1 / 2}} .
$$


For $\chi \bmod q$, define

$$
\begin{aligned}
C_{2}(\chi, a) & =\sum_{h=1}^{q} \bar{\chi}(h) e\left(\frac{a h^{2}}{q}\right), & C(\chi, a) & =\sum_{h=1}^{q} \bar{\chi}(h) e\left(\frac{a h}{q}\right), \\
C_{2}(q, a) & =C_{2}\left(\chi_{0}, a\right), & C(q, a) & =C\left(\chi_{0}, a\right) .
\end{aligned}
$$

Here $\chi_{0}$ is the principal character modulo $q$.

If $\chi_{1}, \chi_{2}, \chi_{3}$ are characters $\bmod q$, then let

$$
B\left(n, q ; \chi_{1}, \chi_{2}, \chi_{3}\right)=\frac{1}{\phi^{3}(q)} \sum_{\substack{a=1 \\(a, q)=1}}^{q} C\left(\chi_{1}, a\right) C_{2}\left(\chi_{2}, a\right) C_{2}\left(\chi_{3}, a\right) e\left(-\frac{a n}{q}\right)
$$

$$
A(n, q)=B\left(n, q ; \chi_{0}, \chi_{0}, \chi_{0}\right), \quad \mathfrak{S}(n, X)=\sum_{q \leq X} A(n, q) .
$$

LEMMA 2 (Lemma 2.1 of [8]). Let $\chi_{j} \bmod r_{j}$ with $j=1,2,3$ be primitive characters, $r_{0}=\left[r_{1}, r_{2}, r_{3}\right]$, and $\chi_{0}$ the principal character mod $q$. Then

$$
\sum_{\substack{q \leq x \\ r_{0} \mid q}}\left|B\left(n, q ; \chi_{1} \chi_{0}, \chi_{2} \chi_{0}, \chi_{3} \chi_{0}\right)\right| \ll r_{0}^{-1 / 2+\varepsilon}(\log x)^{c} .
$$

On the minor arcs, we need estimates for the measure of the set

$$
\mathcal{E}_{\lambda}:=\{\alpha \in(0,1]:|h(\alpha)| \geq \lambda L\} .
$$

The following lemma is due to Heath-Brown and Puchta [2].

Lemma 3. We have

$$
\operatorname{meas}\left(\mathcal{E}_{\lambda}\right) \ll N^{-E(\lambda)} \text { with } \quad E(0.9108)>\frac{19}{24}+10^{-10} .
$$

Proof. Let

$$
\begin{aligned}
T_{h}(\alpha) & =\sum_{0 \leq n \leq h-1} e\left(\alpha 2^{n}\right), \\
F(\xi, h) & =\frac{1}{2^{h}} \sum_{r=0}^{2^{h}-1} \exp \left\{\xi \operatorname{Re}\left(T_{h}\left(r / 2^{h}\right)\right)\right\}, \\
E(\lambda) & =\frac{\xi \lambda}{\log 2}-\frac{\log F(\xi, h)}{h \log 2}-\frac{\varepsilon}{\log 2} .
\end{aligned}
$$

Then for any $\xi, \varepsilon>0$, and any $h \in \mathbb{N}$, we have

$$
\operatorname{meas}\left(\mathcal{E}_{\lambda}\right) \ll N^{-E(\lambda)} \text {. }
$$

This was proved in Section 7 of [2]. Taking $\xi=1.31, h=18$, we get

$$
E(0.9108)>\frac{19}{24}+10^{-10} \text {. }
$$

This completes the proof of the lemma. 
3. The major arcs. Let

$$
\begin{aligned}
f^{*}(\alpha) & =\frac{C_{2}(q, a)}{\phi(q)} \sum_{M<m^{2} \leq N} e\left(\beta m^{2}\right), \\
g^{*}(\alpha) & =\frac{C(q, a)}{\phi(q)} \sum_{M<m \leq N} e(\beta m) .
\end{aligned}
$$

We now proceed to estimate the quantity

$$
\int_{\mathfrak{M}} f^{2}(\alpha) g(\alpha) e(-\alpha n) d \alpha-\int_{\mathfrak{M}} f^{* 2}(\alpha) g^{*}(\alpha) e(-\alpha n) d \alpha,
$$

which we think of as the error of approximation of the integral over $\mathfrak{M}$ by the expected term.

By the standard major arcs techniques we have

$$
\int_{\mathfrak{M}} f^{* 2}(\alpha) g^{*}(\alpha) e(-\alpha n) d \alpha=P_{0} \mathfrak{S}(n, P)(1+o(1)),
$$

where

$$
P_{0}=\pi n / 4,
$$

and $\mathfrak{S}(n, P)$ is defined by $(2.11)$. Define

$$
\begin{aligned}
W(\chi, \beta) & =\sum_{M<p^{2} \leq N}(\log p) \chi(p) e\left(\beta p^{2}\right)-D(\chi) \sum_{M<m^{2} \leq N} e\left(\beta m^{2}\right), \\
W^{\sharp}(\chi, \beta) & =\sum_{M<p \leq N}(\log p) \chi(p) e(\beta p)-D(\chi) \sum_{M<m \leq N} e(\beta m),
\end{aligned}
$$

where $D(\chi)$ is 1 or 0 according as $\chi$ is principal or not.

Just as in $[1,(4.1)]$ we can rewrite $f(\alpha)$ and $g(\alpha)$ as

$$
\begin{aligned}
& f\left(\frac{a}{q}+\beta\right)=\frac{C_{2}(q, a)}{\phi(q)} \sum_{M<m^{2} \leq N} e\left(\beta m^{2}\right)+\frac{1}{\phi(q)} \sum_{\chi \bmod q} C_{2}(\chi, a) W(\chi, \beta), \\
& g\left(\frac{a}{q}+\beta\right)=\frac{C(q, a)}{\phi(q)} \sum_{M<m \leq N} e(\beta m)+\frac{1}{\phi(q)} \sum_{\chi \bmod q} C(\chi, a) W^{\sharp}(\chi, \beta) .
\end{aligned}
$$

So we can use (3.5) and (3.6) to express the difference in (3.2) as a linear combination of error terms involving $f^{*}(\alpha)$ and $g^{*}(\alpha)$, and $W(\chi, \beta)$ and $W^{\sharp}(\chi, \beta)$.

We shall focus on the most troublesome among the error terms that arise, namely the multiple sum

$$
\sum_{q \leq P} \sum_{\chi_{1} \bmod q} \sum_{\chi_{2} \bmod q} \sum_{\chi_{3} \bmod q} B\left(n, q ; \chi_{1}, \chi_{2}, \chi_{3}\right) J\left(n, q, \chi_{1}, \chi_{2}, \chi_{3}\right) .
$$


Here $B\left(n, q ; \chi_{1}, \chi_{2}, \chi_{3}\right)$ is defined in $(2.10)$, and

$$
J\left(n, q, \chi_{1}, \chi_{2}, \chi_{3}\right)=\int_{-1 / q Q}^{1 / q Q} W^{\sharp}\left(\chi_{1}, \beta\right) W\left(\chi_{2}, \beta\right) W\left(\chi_{3}, \beta\right) e(-\beta n) d \beta .
$$

We first reduce (3.7) to a sum over primitive characters. Suppose $\chi_{j}^{*} \bmod r_{j}$ with $r_{j} \mid q$ is the primitive character inducing $\chi_{j}$. In general, if $\chi \bmod q$, $q \leq P$, is induced by a primitive character $\chi^{*} \bmod r$ with $r \mid q$, we have

$$
W^{\sharp}(\chi, \beta)=W^{\sharp}\left(\chi^{*}, \beta\right), \quad W(\chi, \beta)=W\left(\chi^{*}, \beta\right) .
$$

By Cauchy's inequality

$$
J\left(n, q, \chi_{1}, \chi_{2}, \chi_{3}\right) \ll W^{\sharp}\left(\chi_{1}^{*}\right) W\left(\chi_{2}^{*}\right) W\left(\chi_{3}^{*}\right),
$$

where for a character $\chi \bmod r$,

$$
W^{\sharp}(\chi)=\max _{|\beta| \leq 1 / r Q}\left|W^{\sharp}(\chi, \beta)\right|, \quad W(\chi)=\left(\int_{-1 / r Q}^{1 / r Q}|W(\chi, \beta)|^{2} d \beta\right)^{1 / 2} .
$$

Using (3.9) we can bound (3.7) by

$$
\sum_{r_{1} \leq P} \sum_{\chi_{1}}^{*} \sum_{r_{2} \leq P} \sum_{\chi_{2}}^{*} \sum_{r_{3} \leq P} \sum_{\chi_{3}}^{*} W^{\sharp}\left(\chi_{1}\right) W\left(\chi_{2}\right) W\left(\chi_{3}\right) B\left(n, \chi_{1}, \chi_{2}, \chi_{3}\right) .
$$

Here $\sum_{r_{j}} \sum_{\chi}^{*}$ denotes a summation over the primitive characters mod $r_{j}$ $\leq P$, and

$$
B\left(n, \chi_{1}, \chi_{2}, \chi_{3}\right)=\sum_{\substack{q \leq P \\ r_{0} \mid q}}\left|B\left(n, q ; \chi_{1} \chi_{0}, \chi_{2} \chi_{0}, \chi_{3} \chi_{0}\right)\right|,
$$

where $r_{0}=\left[r_{1}, r_{2}, r_{3}\right]$ and $\chi_{0}$ is the principal character $\bmod q$.

By Lemma 2 we have

$$
B\left(n, \chi_{1}, \chi_{2}, \chi_{3}\right) \ll r_{0}^{-1 / 2+\varepsilon} L^{c},
$$

and by [7, Lemma 2.4] we have

$$
\sum_{r \leq R} \sum_{\chi}^{*}[r, d]^{-1 / 2+\varepsilon} W(\chi) \ll d^{-1 / 2+\varepsilon} L^{c}
$$

whenever $R \leq N^{1 / 6-\varepsilon}$. Thus the sixfold sum in (3.11) does not exceed

$$
L^{c} \sum_{r_{1} \leq P} \sum_{\chi_{1}}^{*} r_{1}^{-1 / 2+\varepsilon} W^{\sharp}\left(\chi_{1}\right) .
$$

To estimate $\sum_{r_{1} \leq P} \sum_{\chi_{1}}^{*} r_{1}^{-1 / 2+\varepsilon} W^{\sharp}\left(\chi_{1}\right)$, we can modify the proof of Lemma 2.3 of [7] for $k=1$. For $L^{B}<R \leq P$, where $B$ is a constant depending on $A$, the right-hand sides of (5.1) and (5.2) of [7] should be replaced by 
$N^{1 / 2}\left(T_{1}+1\right)^{1 / 2} L^{-A}$ and $N^{1 / 2} T_{2} L^{-A}$, by using Theorem 4.1 of [7]; moreover, since $R \leq P=N^{1 / 6-\varepsilon}$, we get

$$
\sum_{L^{B}<r_{1} \leq P} \sum_{\chi_{1}}^{*} r_{1}^{-1 / 2+\varepsilon} W^{\sharp}\left(\chi_{1}\right) \ll N L^{-A} \quad \text { for any } A>0 .
$$

For the case $R \leq L^{B}$, in the same way as in [7] we deduce that

$$
\sum_{r_{1} \leq L^{B}} \sum_{\chi_{1}}^{*} r_{1}^{-1 / 2+\varepsilon} W^{\sharp}\left(\chi_{1}\right) \ll N L^{-A} \quad \text { for any } A>0 .
$$

We have shown that the sum in (3.7) is $O\left(N L^{-A}\right)$ for any fixed $A>0$. Recall that (3.7) was one of several error terms in a representation of (3.2). Since the other error terms in that representation can be estimated similarly, we conclude that the difference in (3.2) is $O\left(N L^{-A}\right)$.

Together with (3.3) we obtain the following result:

Lemma 4. For all integers $n \in \mathcal{A}$, we have

$$
\int_{\mathfrak{M}} f^{2}(\alpha) g(\alpha) e(-\alpha n) d \alpha=(\pi / 4+o(1)) \mathfrak{S}(n, P) n+O(N / \log N) .
$$

Lemma 5. For $n \in \mathcal{A}$, we have

$$
\mathfrak{S}(n, P) \geq 2.27473966 \text {. }
$$

Otherwise, we have $\mathfrak{S}(n, P)=O\left(P^{-1+\varepsilon}\right)$.

Proof. By [8, p. 114], we have

$$
\mathfrak{S}(n, P)=\sum_{q=1}^{\infty} A(n, q)+O\left(P^{-1+\varepsilon}\right) .
$$

By $[10,(3.14)]$, when $(a, q)=1$, we have $C(q, a)=\mu(q)$. Hence

$$
A(n, q)=\frac{\mu(q)}{\phi^{3}(q)} \sum_{\substack{a=1 \\(a, q)=1}}^{q} C_{2}^{2}(q, a) e\left(-\frac{a n}{q}\right)
$$

and for $k \geq 2, A\left(n, p^{k}\right)=0$. Since $A(n, q)$ is multiplicative, we have

$$
\mathfrak{S}(n, P)=\prod_{p=2}^{\infty}(1+A(n, p))+O\left(P^{-1+\varepsilon}\right) .
$$

By direct computation, for $n \in \mathcal{A}$ we have

$$
1+A(n, 2)=2, \quad 1+A(n, 3)=3 / 2 .
$$

If $n \equiv 0(\bmod 2)$, we have $1+A(n, 2)=0$. When $n \equiv 2(\bmod 3)$, we have 
$1+A(n, 3)=0$. By $[8$, p. 114], for $p \geq 5$, we have

$$
1+A(n, p) \geq \begin{cases}1-\frac{p+1}{(p-1)^{3}}, & p \equiv 1(\bmod 4), \\ 1-\frac{3 p-1}{(p-1)^{3}}, & p \equiv-1(\bmod 4) .\end{cases}
$$

Hence

$$
\prod_{p \geq 5}(1+A(n, p)) \geq \prod_{p \equiv 1(\bmod 4)}\left(1-\frac{p+1}{p \geq 5}\right) \prod_{\substack{p \equiv-1(\bmod 4) \\ p \geq 5}}\left(1-\frac{3 p-1}{(p-1)^{3}}\right) .
$$

By the elementary inequality

$$
(1+x)^{a}<1+a x+\frac{a(a-1)}{2} x^{2} \quad \text { if } a>2,-1<x<0,
$$

for $p>82$ and $p \equiv 1(\bmod 4)$ we have

$$
1-\frac{p+1}{(p-1)^{3}} \geq\left(1-\frac{1}{(p-1)^{2}}\right)^{3.025},
$$

and for $p>82$ and $p \equiv-1(\bmod 4)$,

$$
1-\frac{3 p-1}{(p-1)^{3}} \geq\left(1-\frac{1}{(p-1)^{2}}\right)^{3.025} .
$$

Thus

$$
\begin{aligned}
& \prod_{p \geq 5}(1+A(n, p)) \\
& \geq \prod_{\substack{p \equiv 1(\bmod 4) \\
5 \leq p<82}}\left(1-\frac{p+1}{(p-1)^{3}}\right) \prod_{\substack{p \equiv-1(\bmod 4) \\
5 \leq p<82}}\left(1-\frac{3 p-1}{(p-1)^{3}}\right) \prod_{p>82}\left(1-\frac{1}{(p-1)^{2}}\right)^{3.025} \\
& =\prod_{\substack{p \equiv 1(\bmod 4) \\
5 \leq p<82}}\left(1-\frac{p+1}{(p-1)^{3}}\right) \prod_{\substack{p \equiv-1(\bmod 4) \\
5 \leq p<82}}\left(1-\frac{3 p-1}{(p-1)^{3}}\right) \\
& \quad \times \prod_{p \equiv 1(\bmod 4)}\left(1-\frac{1}{(p-1)^{2}}\right)^{-3.025} \underset{\substack{5 \leq p<82 \\
p \equiv-1(\bmod 4)}}{3 \leq p<82}\left(1-\frac{1}{(p-1)^{2}}\right)^{-3.025} \\
& \quad \times \prod_{p \geq 3}\left(1-\frac{1}{(p-1)^{2}}\right)^{3.025} \geq 1.11571 \cdot\left(0.6601 \cdot \frac{4}{3}\right)^{3.025}>0.7582465536,
\end{aligned}
$$

where we have used the well known result $\prod_{p \geq 3}\left(1-1 /(p-1)^{2}\right)=0.6601 \ldots$. By (3.14), (3.15) and the above estimate, we get the lemma. 
4. Proof of Theorem. We need the following lemmas.

Lemma 6. Let $\mathcal{A}(N, k)=\left\{n \geq 2: n=N-2^{\nu_{1}}-\cdots-2^{\nu_{k}}\right\}$ with $k \geq 100$. Then for odd $N$, we have

$$
\sum_{\substack{n \in \mathcal{A}(N, k) \\ n \neq 2(\bmod 3)}} n \geq\left(2 / 3-2^{-90}\right) N L^{k}
$$

Proof. Let $((\nu))$ mean that $\nu_{1}, \ldots, \nu_{k}$ satisfies

$$
1 \leq \nu_{1}, \ldots, \nu_{k} \leq \log _{2}(N / k L), \quad N-2^{\nu_{1}}-\cdots-2^{\nu_{k}} \equiv 0(\bmod 3) .
$$

Then $n \geq N-N / L$, and

$$
\sum_{\substack{n \in \mathcal{A}(N, k) \\ n \equiv 0(\bmod 3)}} n \geq \sum_{((\nu))}\left(N-2^{\nu_{1}}-\cdots-2^{\nu_{k}}\right) \geq\left(N-\frac{N}{L}\right) \sum_{((\nu))} 1 .
$$

For odd $q$, let $\varepsilon(q)$ be the order of 2 in the multiplicative group of integers modulo $q$. Let

$$
H(d, N, K)=\sharp\left\{\left(\nu_{1}, \ldots, \nu_{K}\right): 1 \leq \nu_{i} \leq \varepsilon(d), d \mid N-\sum 2^{\nu_{i}}\right\} .
$$

When $d=3, \varepsilon(3)=2$, and it is an easy exercise to check that

$$
H(3, N, K)= \begin{cases}\frac{1}{3}\left(2^{K}-(-1)^{K}\right), & 3 \nmid N, \\ \frac{1}{3}\left(2^{K}+(-1)^{K}\right), & 3 \mid N .\end{cases}
$$

Thus if $K>100$ we have

$$
H(3, N, K) \varepsilon(3)^{-K} \geq \frac{1}{3}\left(1-2^{-98}\right),
$$

and

$$
\sum_{((\nu))} 1 \geq H(3, N, k)\left(\left[\log _{2}(N / k L) / \varepsilon(3)\right]-2\right)^{k} \geq \frac{1}{3}\left(1-2^{-96}\right) L^{k} .
$$

Hence

$$
\sum_{\substack{n \in \mathcal{A}(N, k) \\ n \equiv 0(\bmod 3)}} n \geq\left(1 / 3-2^{-95}\right) N L^{k}
$$

Similarly,

$$
\sum_{\substack{n \in \mathcal{A}(N, k) \\ n \equiv 1(\bmod 3)}} n \geq\left(1 / 3-2^{-95}\right) N L^{k}
$$

From this and (4.3) we get the lemma. 
Lemma 7 (Lemma 3 of [5]). Let $f(\alpha)$ and $h(\alpha)$ be as in (2.4). Then

$$
\int_{0}^{1}|f(\alpha) h(\alpha)|^{4} d \alpha \leq c_{1} \frac{\pi^{2}}{16} N L^{4},
$$

where

$$
c_{1} \leq\left(\frac{32^{4} \cdot 101 \cdot 1.620767}{3}+\frac{8 \cdot \log ^{2} 2}{\pi^{2}}\right)(1+\varepsilon)^{9} .
$$

Lemma 8. Let $g(\alpha)$ and $h(\alpha)$ be as in (2.4). Then

$$
\int_{\mathfrak{m}}|g(\alpha) h(\alpha)|^{2} d \alpha \leq 12.3685 c_{0} N L^{2},
$$

where

$$
c_{0}=\prod_{p \geq 3}\left(1-\frac{1}{(p-1)^{2}}\right)=0.6601 \ldots
$$

Proof. This is actually Lemma 10 of [2]. By (8.14) of [9], we can replace (41) of [2] by $C_{2} \leq 1.94$, and then by the proof of Lemma 9 of [2] the assertion follows.

Now we prove the Theorem. Let $\mathcal{E}_{\lambda}$ be as defined in (2.12), and $\mathfrak{M}$ and $\mathfrak{m}$ as in (2.6) and (2.7) with $P, Q$ determined in (2.2). Then (2.3) becomes

$$
R(N)=\int_{0}^{1} f^{2}(\alpha) g(\alpha) h^{k}(\alpha) e(-\alpha N) d \alpha=\int_{\mathfrak{M}}+\int_{\mathfrak{m} \cap \mathcal{E}_{\lambda}}+\int_{\mathfrak{m} \backslash \mathcal{E}_{\lambda}} .
$$

For the major arcs, by Lemma 4 we have

$$
\begin{aligned}
\int_{\mathfrak{M}} f^{2}(\alpha) g(\alpha) & h^{k}(\alpha) e(-\alpha N) d \alpha \\
& =\sum_{n \in \mathcal{A}(N, k)} \int_{\mathfrak{M}} f^{2}(\alpha) g(\alpha) e(-\alpha n) d \alpha \\
& =\left(\frac{\pi}{4}+o(1)\right) \sum_{n \in \mathcal{A}(N, k)} \mathfrak{S}(n, P) n+O\left(N L^{k-1}\right) \\
& \geq 2.27473966\left(\frac{\pi}{4}+o(1)\right)\left\{\sum_{\substack{n \in \mathcal{A}(N, k) \\
n \neq \equiv(\bmod 3)}} n\right\}+O\left(N L^{k-1}\right) \\
& \geq 1.516492 \frac{\pi}{4} N L^{k},
\end{aligned}
$$

where we have used Lemmas 5 and 6. 
For the second integral in (4.5), by Dirichlet's lemma on rational approximation, any $\alpha \in \mathfrak{m}$ can be written as

$$
\alpha=\frac{a}{q}+\beta, \quad|\beta| \leq \frac{1}{q N^{3 / 4}},
$$

for some integers $a, q$ with $1 \leq a \leq q \leq N^{3 / 4},(a, q)=1$. If $q \leq P=N^{1 / 6-\varepsilon}$, since $\alpha \in \mathfrak{m}$, we have $P L^{10}<N|q \alpha-a|$; otherwise we have $q>P$; hence $q+N|q \alpha-a|>P$ for any $\alpha \in \mathfrak{m}$. By Lemma 1,

$$
\max _{\alpha \in \mathfrak{m}}|f(\alpha)| \ll N^{1 / 2-1 / 16+\varepsilon} .
$$

By Theorem 3.1 of Vaughan [10],

$$
\max _{\alpha \in \mathfrak{m}}|g(\alpha)| \ll N^{1-1 / 12+\varepsilon} .
$$

Therefore

$$
\int_{\mathfrak{m} \cap \mathcal{E}_{\lambda}} \ll N^{-E(0.9108)} N^{2-5 / 24+\varepsilon} L^{k} \ll N^{1-\varepsilon},
$$

where we have used Lemma 3 for $\lambda=0.9108$.

For the last integral in (4.5), with the definition of $\mathcal{E}_{\lambda}$, and Lemmas 7 and 8 , by Cauchy's inequality we have

$$
\begin{aligned}
\int_{\mathfrak{m} \backslash \mathcal{E}_{\lambda}} & \leq(\lambda L)^{k-3}\left(\int_{0}^{1}|f(\alpha) h(\alpha)|^{4} d \alpha\right)^{1 / 2}\left(\int_{\mathfrak{m}}|g(\alpha) h(\alpha)|^{2} d \alpha\right)^{1 / 2} \\
& \leq 21616 \lambda^{k-3} \frac{\pi}{4} N L^{k} .
\end{aligned}
$$

Combining this with (4.6) and (4.8), we get

$$
R(N) \geq \frac{\pi}{4} N L^{k}\left(1.516492-21616 \lambda^{k-3}\right) .
$$

When $k \geq 106$, for $\lambda=0.9108$, by the above estimate we have

$$
R(N)>0 \text {. }
$$

This means that every large odd integer $N$ can be written in the form of (1.2) for $k \geq 106$.

Acknowledgments. Thanks for the referee's comments. This work was supported by the National Natural Science Foundation of China (Grant No. 10471090).

\section{References}

[1] G. Harman and A. V. Kumchev, On sums of squares of primes, Math. Proc. Cambridge Philos. Soc. 140 (2006), 1-13.

[2] D. R. Heath-Brown and J. C. Puchta, Integers represented as a sum of primes and powers of two, Asian J. Math. 6 (2002), 535-565. 
[3] L. K. Hua, Some results in additive prime number theory, Quart. J. Math. Oxford 9 (1938), 68-80.

[4] A. V. Kumchev, On Weyl sums over primes and almost primes, Michigan Math. J. 54 (2006), 243-268.

[5] H. Z. Li, Four prime squares and powers of 2, Acta Arith. 125 (2006), 383-391.

[6] J. Y. Liu, M. C. Liu and T. Zhan, Squares of primes and powers of 2, Monatsh. Math. 128 (1999), 283-313.

[7] J. Y. Liu and T. Zhan, The exceptional set in Hua's theorem for three squares of primes, Acta Math. Sin. (Engl. Ser.) 21 (2005), 335-350.

[8] T. Liu, Representation of odd integers as the sum of one prime, two squares of primes and powers of 2, Acta Arith. 115 (2004), 97-118.

[9] J. Pintz and I. Z. Ruzsa, On Linnik's approximation to Goldbach's problem, I, ibid. 109 (2003), 169-194.

[10] R. C. Vaughan, The Hardy-Littlewood Method, Cambridge Univ. Press, 1981.

Department of Mathematics

Shanghai Jiaotong University

Shanghai 200240

People's Republic of China

E-mail: lihz@sjtu.edu.cn

Received on 21.9.2006

and in revised form on 11.1.2007 